\title{
ADVANCES IN AUTOMATIC EBSP SINGLE ORIENTATION MEASUREMENTS
}

\author{
K. KUNZE, ${ }^{\text {a }}$ S. I. WRIGHT, ${ }^{\text {b }}$ B. L. ADAMS ${ }^{a}$ and D. J. DINGLEY $\dagger$ \\ Department of Mechanical Engineering, Yale University, New Haven, CT \\ $\dagger$ H. H. Wills Physics Laboratory, University of Bristol, Bristol, England
}

\begin{abstract}
A completely automated system which couples automatic analysis of electron backscatter diffraction patterns (EBSPs) with precise movement of the sample in the SEM is described. The Hough transform is implemented into an existing technique for identifying lattice orientation through automatic analysis of EBSPs. The ability of the Hough transform approach to correctly identify diffraction bands is quantitatively compared with the Burns algorithm. Both methods were tested on 1000 EBSPs from well annealed oxygen-free, electrical grade (OFE) copper, 120 patterns from as-cast commercially pure aluminum and 106 patterns from $40 \%$ channel-die compressed aluminum. Only slight differences were found in the ability of the methods to correctly identify bands in the sample EBSPs. The three test runs resulted in 95-99\% (OFE $\mathrm{Cu}$ ), 97.5-99\% (as-cast $\mathrm{Al}$ ) and $81-85 \%$ (deformed Al) correctly determined orientations (less than $5^{\circ}$ off the manually determined reference orientations). The limits in the ability of the band detection algorithms to fix the bands exactly are discussed. To obtain the orientation which satisfies the detected bands most completely, a method of averaging solutions obtained from different triplets of bands is introduced.
\end{abstract}

KEY WORDS EBSP, electron diffraction, single orientation measurements.

\section{INTRODUCTION}

The need for coupling morphological and orientational aspects of microstructure together into more complete characterizations of microstructure is well established. Until now, examinations of spatial orientation relationships have been limited to measurements from small regions within planar sections. In order to determine statistically significant relationships, single orientation measurements made at very many points are needed. When many measurements are collected on several oblique section planes a statistical three dimensional picture of the arrangement of grains and their orientations can be constructed using stereology. Wright and Adams (1992) have shown that a reliable rapid determination of lattice orientation can be achieved through automatic analysis of electron backscatter diffraction patterns (EBSPs). (For background on the use of EBSPs in the SEM for determinining lattice orientation see Venables and Harland (1973) and Dingley and Baba-Kishi (1986).) In order to remove the direct need of an operator for collecting single orientation measurements, a fully automated system requires the computer to not only analyze the diffraction patterns but also to control the position of the sample with reference to the electron beam.

\footnotetext{
${ }^{a}$ Present address: Brigham Young University, Dept. Manufact. Engineering, 435 CTB, Provo, UT 84602.

${ }^{b}$ Present address: Los Alamos National Laboratory, Los Alamos, NM 87545.
} 
This paper describes advances in hardware and software for achieving a reliable fully automated system for collecting single orientation measurements. Additions to the method proposed by Lassen, Conradsen and Juul-Jensen (1992) based on the Hough image transform for detecting bands in the diffraction patterns are described. A method of averaging orientations using quaternions to find the best fit to all detected bands is given. Results of automatic indexing using the Hough transform method and the method of Wright and Adams (1992) based on the Burns algorithm for detecting straight edges (see Burns, Hanson and Riseman (1986)) are presented for three sets of EBSPs: 1000 patterns from well annealed oxygen-free electrical grade (OFE) copper, 120 patterns from commercially pure as-cast aluminum and 106 patterns from $40 \%$ channel-die compressed commercially pure aluminum. Finally, the capabilities of the two band detection methods (denoted throughout as the Hough and Burns techniques) are discussed and compared.

\section{HARDWARE}

The current hardware configuration is shown in Figure 1 and described as follows. In order to attain high quality EBSPs, a high gain fiber optic camera is used to record video images. Using fiber optics removes any image distortions inherent to conventional camera lenses. The video images are processed by the camera control unit. The control unit is capable of averaging 64 video frames and subtracting a background. A background image is obtained when the SEM is in image mode. The sample holder is fixed on a piezoelectric $x-y$ stage, which is mounted at an angle of $70^{\circ}$ to the horizontal in the SEM vacuum chamber. Since the specimen surface is aligned parallel to the plane of stage movement (both tilted by $70^{\circ}$ ), translations of the sample within this plane keep the electron beam in focus. Small variations in the surface morphology do not influence the

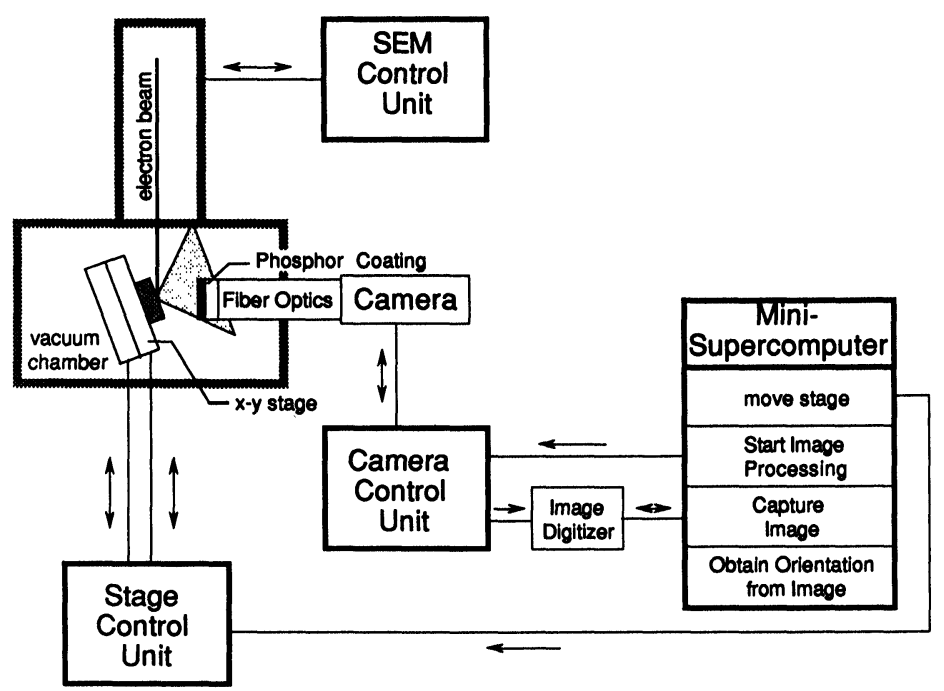

Figure 1 Schematic of automatic single orientation measurement system. 
calibrated beam geometry significantly. The smallest possible step size is $0.1 \mu \mathrm{m}$ and the total range of travel is $27 \mathrm{~mm} \times 26 \mathrm{~mm}$. The reproducibility over the entire range of travel is $0.25 \mu \mathrm{m}$. The camera control unit and the stage control unit are interfaced to a mini-super computer; thus, the computer becomes the central control unit for both the stage and the video camera unit. The computer has four processors and much of the code has been parallelized to take advantage of the parallel architecture. The computer has a frame capture board installed to capture the video images from the camera control unit.

Currently, the general method used is to make lattice orientation measurements on a grid, however, an irregular array of points can also be easily programmed into the computer. The computer controls the procedure in the following manner: first, it tells the stage to move to a given $(x, y)$ position; second, it tells the camera control unit to process an image; third, it captures the processed image through the video digitizer board and fourth, it analyzes the pattern and records the corresponding Euler angles, the $(x, y)$ position and an image quality parameter. This procedure is repeated for all points on the grid. Using our current pattern analysis software, we are capable of measuring approximately 900 orientations an hour with no direct operator intervention.

\section{BAND ANALYSIS USING HOUGH TRANSFORM}

The first step in an automated analysis of electron backscatter patterns consists of detecting the diffraction bands. The Burns algorithm as applied by Wright and Adams (1992) looks locally for lines of high contrast gradient and thus for edges of the bands, which are then combined to form global bands by finding pairs of opposite edges. A different approach, recently proposed by Lassen, Conradsen and Juul-Jensen (1992), incorporates the Hough transform to detect directly global stripes of higher image intensity. With some further improvements of this method we obtained an algorithm for band detection, which compares well to the already established Burns technique.

The Hough transform (see survey by Illingworth and Kittler, 1988) has been applied in a variety of ways to detect complex patterns in digital images. Once the shape of an object is described by an appropriate set of parameters, one looks for the most probable parameter values. In this way a global detection problem in the original image is transferred into a local peak search problem in the parameter space.

The normal parametrization for line detection is given by

$$
\rho_{i}=x_{k} \cos \theta_{i}+y_{k} \sin \theta_{i}
$$

where $\left(x_{k}, y_{k}\right)$ are the coordinates of a pixel in the original image, and $\left(\rho_{i}, \theta_{i}\right)$ are the parameters of a straight line in the image according to Figure 2. A fixed image point $\left(x_{k}, y_{k}\right)$ corresponds to a sinusoidal curve in $(\rho, \theta)$ space. On the other hand, a fixed parameter set $\left(\rho_{i}, \theta_{i}\right)$ characterizes all image points on a straight line in $(x, y)$ coordinates. All the sinusoidal curves, which correspond to collinear $(x, y)$ points, intersect each other in the parameter point $\left(\rho_{i}, \theta_{i}\right)$. The objective is to find those parameters which correspond to lines of high intensity in the $(x, y)$ image. 


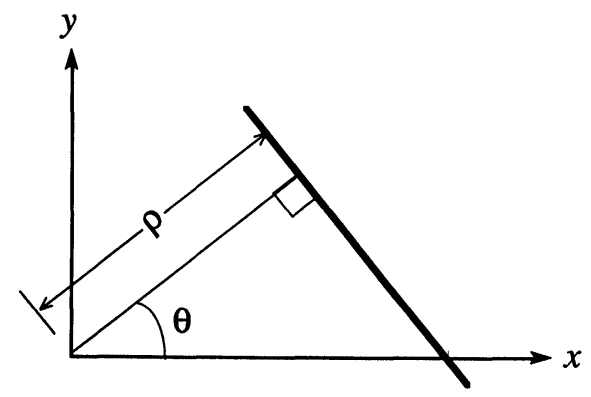

Figure 2 Definition of Hough Parameters.

The bounded parameter regions are divided into finite numbers of small intervals, for which an accumulator array $H\left(\rho_{i}, \theta_{i}\right)$ is defined. As described by Lassen, Conradsen and Juul-Jensen (1992), the image intensity $I\left(x_{k}, y_{k}\right)$ of each pixel is added to every cell $H\left(\rho_{i}, \theta_{i}\right)$ along the curve (Eq. (1)) and gives a vote for all lines through the point $\left(x_{k}, y_{k}\right)$. Then points of high values in the Hough array represent lines of high image intensity in the pattern.

A characteristic profile appears in the Hough transform of the original image of $100 \times 100$ pixels by the procedure followed by Lassen, Conradsen and JuulJensen (1992). This background structure arises from the square shape of the image array and its value at $\left(\rho_{i}, \theta_{i}\right)$ is proportional to the length of that line within the image. It can be obtained by the transform of a homogeneous grey image. Since the true peaks are small compared to these variations in the background intensity, they may be over- or underestimated in dependence on their position in Hough space. Artificial peaks due to the background profile (e.g. at $\left(\rho= \pm 50, \theta=0^{\circ}, 90^{\circ}, 180^{\circ}\right)$ or at $\left.\left(\rho=0, \theta=45^{\circ}, 135^{\circ}\right)\right)$ may lead to misinterpretations of overlapping peaks in their neighborhood.

In order to eliminate these influences one could normalize the Hough intensities by background subtraction or division. We used two steps to equalize the Hough levels:

1. A circular image array was cut out of the original array of $100 \times 100$ pixels. Then the Hough background only depends monotonically on $\rho$ and does not possess points of large intensity gradients.

2. A constant equal to the average image intensity was subtracted from all pixel values. A homogeneous grey image would then give a Hough transform with zeros at all points. This is equivalent to the subtraction of a background distribution in Hough space, but it is numerically more efficient.

In this manner, all lines of higher image intensity correspond to equally weighted peaks in the Hough array regardless of their position.

The finite width of EBSP bands leads to a certain spreading of the corresponding Hough peaks with a characteristic butterfly shape. According to Leavers and Boyce (1987) the spread in the $\rho$-direction is proportional to the band width. For peak enhancement the Hough array is filtered by a convolution mask of $13 \times 13$ pixels as proposed by Lassen, Conradsen and Juul-Jensen (1992). While the optimum design of the mask depends on the width and profile of the bands, their proposed mask gave satisfactory results especially for relatively weak and narrow 
bands. Since the band width is determined by geometrical settings of the experimental equipment as well as crystallographic parameters of the material under investigation, a dynamical adaptation of this mask would be desirable; however, this would be difficult to implement.

Finally the peaks with the highest Hough intensities are found by a search for local maxima, which only considers nearest neighbors. Three parameters were introduced to control this search:

a) the maximum number of peaks to be found (current setting: 7),

b) the minimum Hough peak intensity (1 in relative units),

c) the minimum distance of neighboring peaks (25 Hough pixels in $\rho$ and $\theta$ ).

In some special cases (e.g. $\langle 100\rangle$ type pole in the pattern center for fcc) relative bandwidths are used to uniquely index the pattern. These can be determined from the spread of the peaks in $\rho$-direction. We found that the distance between the two nearest Hough minima along the same $\theta=$ constant line, normalized corresponding to our settings by a factor of 0.6 , gave a good estimate for the band width. Figure 4 shows the processed Hough transform of a sample image (Figure 3) with crosses at the detected peaks and their corresponding band widths. High quality patterns show the more complex profile of Kikuchi bands, which exhibit a dark and bright edge. This causes a small systematic shift of the detected centerlines towards the bright side, as can be observed in Figure 3.

Whereas the spread of a Hough peak is geometrically determined as described above, the peak magnitude gives a measure of the intensity contrast of that band to its surrounding and can serve as a image quality parameter. For this purpose the parameter $\mathrm{IQ}_{\mathrm{H}}$ has been defined as the sum of the peak magnitudes of all

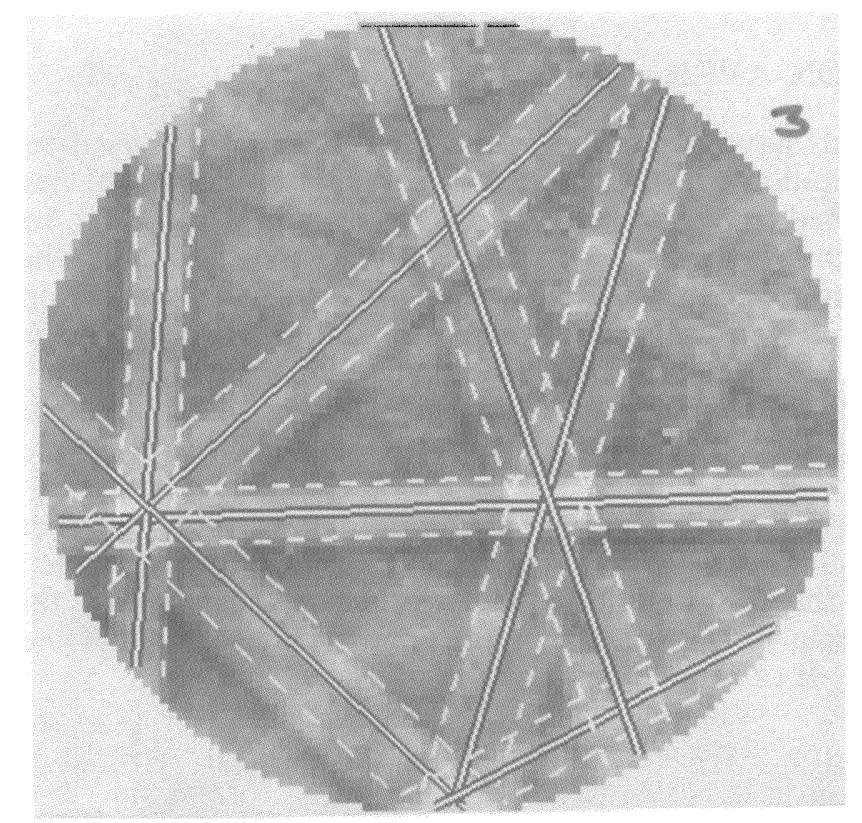

Figure 3 Sample image with detected bands. 


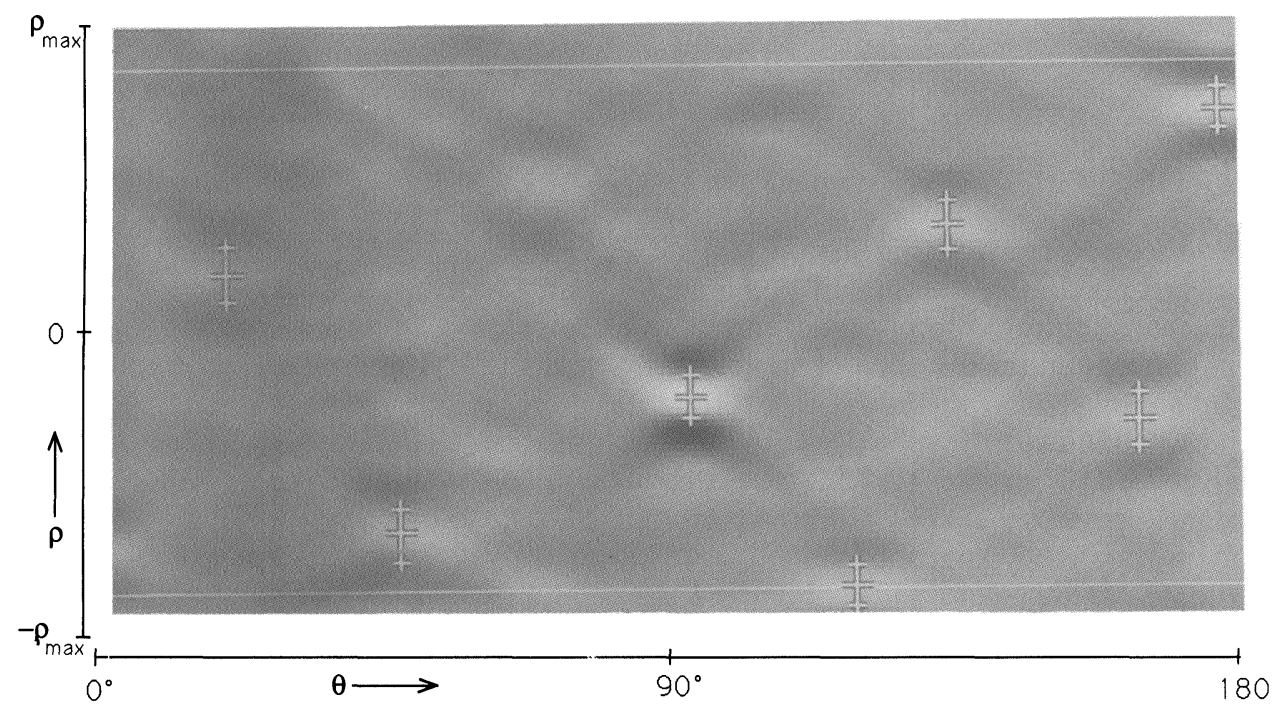

Figure 4 Processed Hough Transform of a circular image indicating the selected peaks and their spreading.

detected peaks. It is zero for a homogeneous grey pattern and limited by the minimum peak intensity (b) as well as by the maximum number of peaks (a). Since at least 3 bands are necessary to uniquely index a pattern, patterns with $\mathrm{IQ}_{\mathrm{H}}<3$ do not yield any solutions.

\section{ORIENTATION AVERAGING}

Once the bands have been detected, a cluster analysis is used to find the most probable orientation out of the set of all possible solutions. The first two steps are already described by Wright and Adams (1992) and work in the following way:

A. For each triplet of bands all possible sets of $(h k l)$, whose theoretical interplanar angles and handedness match those calculated from the detected bands within a given range, are found. Optionally a check can be made to see how well the experimental bandwidth angles fit the theoretical ones. After a sorting algorithm to insure that each member of this set is unique, the corresponding lattice orientation associated with each $(h k l)$ triplet is calculated and stored.

B. All possible solutions obtained from all band triplets are then grouped into clusters of similar orientations. Two orientations are considered to be in the same group, when the misorientation angle between them is less than the pre-set interplanar angle tolerance. The misorientation angle $\omega_{12}$ between the orientations $g_{1}$ and $g_{2}$ is calculated by

$$
\cos \omega_{12}=\frac{1}{2}\left(\max _{j=1, J}\left[\operatorname{trace}\left(g_{c}(j) g_{1} g_{2}^{\top}\right)\right]-1\right)
$$

where $g_{c}(j)(j=1, \ldots, J=24$ for cubic symmetry) stands for all crystal symmetry elements. 
Each cluster within this set of all possible solutions is given three ranking criterion labels: first the number of solutions which belong to this cluster, second the cumulative ranking of the bands used to calculate these orientations and third the number of times the experimental bandwidth angle labels match the theoretical ones. The clusters are then ranked according to their first labels, any ties are broken by ranking according to their second labels, any further ties are broken by ranking according to their third labels.

C. Finally, an average orientation representative for the most probable cluster of solutions is calculated. For this the quaternion representation of orientations is used, which turns out to be the Euclidean description of rotations equivalent to the characterization of directions by unit length vectors $\mathbf{r}=(x, y, z)$.

Each quaternion $\mathbf{Q}=\left(q_{0}, q_{1}, q_{2}, q_{3}\right)$ consists of a scalar $q_{0}$ (with $\left.q_{0} \geq 0\right)$ and a vector $\mathbf{q}=\left(q_{1}, q_{2}, q_{3}\right)$ and represents a point on the upper half of the unit sphere in four-dimensional Euclidean space, satisfying the normalization condition

$$
|\mathbf{Q}|=\left[q_{0}^{2}+q_{1}^{2}+q_{2}^{2}+q_{3}^{2}\right]^{1 / 2}=1
$$

Its coordinates are one-to-one related to the rotation angle $\omega$ and rotation axis $\mathbf{n}$ by

$$
q_{0}=\cos \frac{\omega}{2}, \quad \mathbf{q}=\mathbf{n} \sin \frac{\omega}{2} .
$$

Some properties of quaternions and their connection to Euler angles were recently reviewed by Morawiec and Pospiech (1989) and discussed in terms of ODF representations by Kunze (1991).

The misorientation angle $\omega_{12}$ between two orientations $g(1)$ and $g(2)$ is simply given by the dot product of their corresponding quaternions $\mathbf{Q}(1)$ and $\mathbf{Q}(2)$ according to

$$
\cos \frac{\omega_{12}}{2}=\mathbf{Q}(1) \cdot \mathbf{Q}(2)=\sum_{\mu=0}^{3} q_{\mu}(1) q_{\mu}(2) .
$$

Therefore, the normalized arithmetic mean of $M$ quaternions

$$
\overline{\mathbf{Q}}=N \sum_{m=1}^{M} \mathbf{Q}(m), \quad|\overline{\mathbf{Q}}|=1,
$$

where $N$ is the normalization factor, maximizes the mean dot product between $\overline{\mathbf{Q}}$ and the $\mathbf{Q}(m)$. It gives, at least for small $\omega_{12}$, a good estimate for an average orientation at the center of gravity of the cluster. Indeed, in the second order approximation (with $\cos (\omega / 2) \cong 1-\omega^{2} / 8$ ) the average $\overline{\mathbf{Q}}$ minimizes the mean of the squares of all misorientation angles between $\overline{\mathbf{Q}}$ and the $\mathbf{Q}(m)$.

The outlined algorithm works in the following steps:

1. Calculate $\mathbf{Q}(m)$ from the orientation matrices $g_{j k}(m)$ by

$$
\begin{gathered}
q_{0}=\frac{1}{2}\left[\sum_{i=1}^{3} g_{i i}+1\right]^{1 / 2} \\
q_{i}=\left\{\begin{array}{lll}
\frac{1}{4 q_{0}} \sum_{j, k=1}^{3} \varepsilon_{i j k} g_{j k}, & \text { if } & q_{0}>0 \\
{\left[\frac{g_{i i}+1}{2}\right]^{1 / 2},} & \text { if } & q_{0}=0
\end{array}\right.
\end{gathered}
$$

where $i=1,2,3$. 
2. Calculate average $\overline{\mathbf{Q}}$ and normalize according to Eq. (6).

3. Determine corresponding orientation matrix $\bar{g}$ by

$$
\bar{g}_{i j}=\left(q_{0}^{2}-\mathbf{q}^{2}\right) \delta_{i j}+2 q_{i} q_{j}+2 q_{0} \sum_{k=1}^{3} \varepsilon_{i j k} q_{k} .
$$

\section{EXPERIMENTAL PROCEDURE}

Three sets of EBSPs were obtained from three different samples. The first was a set of one thousand patterns obtained from oxygen-free electrical grade (OFE) copper as previously described in Wright and Adams (1992). These were collected by manually selecting grains which gave good quality patterns. Two other data sets were taken without any biasing toward high quality patterns. One set of data was obtained from a sample of as-cast aluminum ingot and the other from aluminum ingot which had undergone $40 \%$ channel die compression. 120 EBSPs were obtained from the as-cast aluminum on a hexagonal grid of $300 \mu \mathrm{m}$ spacing. The average grain diameter was $\approx 200 \mu \mathrm{m}$. 106 EBSPs were collected on a similar grid on a sample from the deformed aluminum. All of the samples were mechanically polished and then electropolished.

The ESBPs were stored on the computer and indexed manually (by two different people independently in the case of the as-cast $\mathrm{Al}$ ) and then automatically using the Burns Algorithm described in Wright and Adams (1992) and the Hough Algorithm as previously described.

\section{RESULTS}

\section{Copper}

The copper patterns were analyzed incorporating the averaging scheme described earlier and also without the averaging. The averaging improves the precision slightly as can be seen in Figure 5. It does not improve the number of "correct" (i.e. misorientation angle $<5^{\circ}$ ) indexings but makes the "correct" solutions more precise. For example, in both algorithms there were a larger number of patterns which were indexed to within $2^{\circ}$ of the manual solution in the averaged case than in the non-averaged case.

\section{Aluminum}

As expected the deformed aluminum produced much lower quality EBSPs. The larger portion of patterns indexed incorrectly in the bars representing the deformed results in Figure 6 is a direct result of the loss of quality in the EBSPs due to deformation. In fact, some of the patterns contained only one discernable diffraction band. Thus, the measurement is more of a guess than an exact determination. However, it should also be noted in Table 1 that more than $80 \%$ of the patterns were correctly indexed. One feature to note in Figure 6 is that the Hough algorithm appears to be more robust for analyzing poor quality patterns, whereas the Burns algorithm does a better job in the higher quality patterns. This will be discussed in more detail in the following section. 


\section{Annealed Copper}

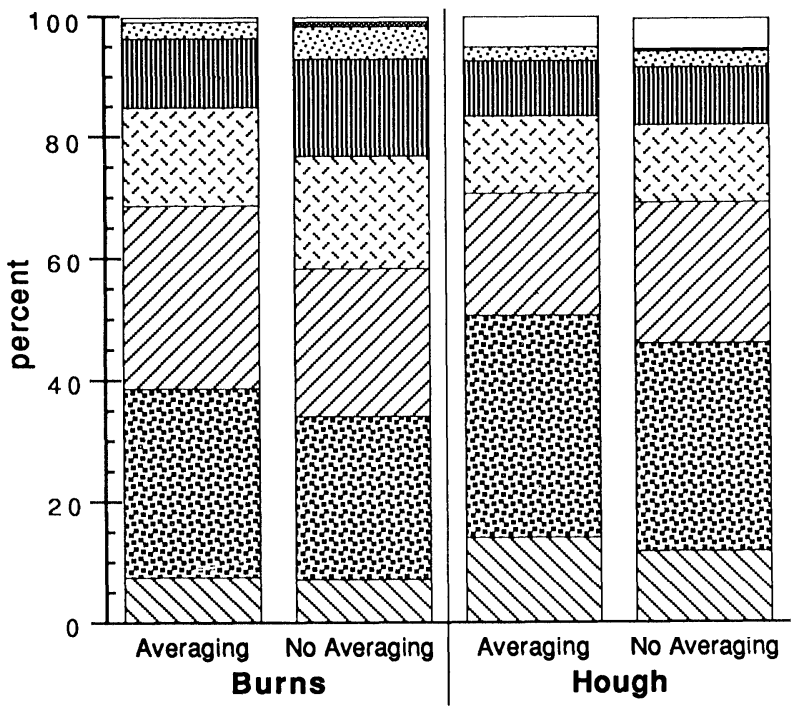

No Solution

$\square>5^{\circ}$

$5^{\circ}$

$4^{\circ}$

IIIII) $3^{\circ}$

2. $2^{\circ}$

D $1.5^{\circ}$

䧄 $1.0^{\circ}$

$\Delta 0.5^{\circ}$

Figure 5 Orientation difference angle of different automatic indexing methods with reference to manual indexing of 1000 patterns from well annealed copper.

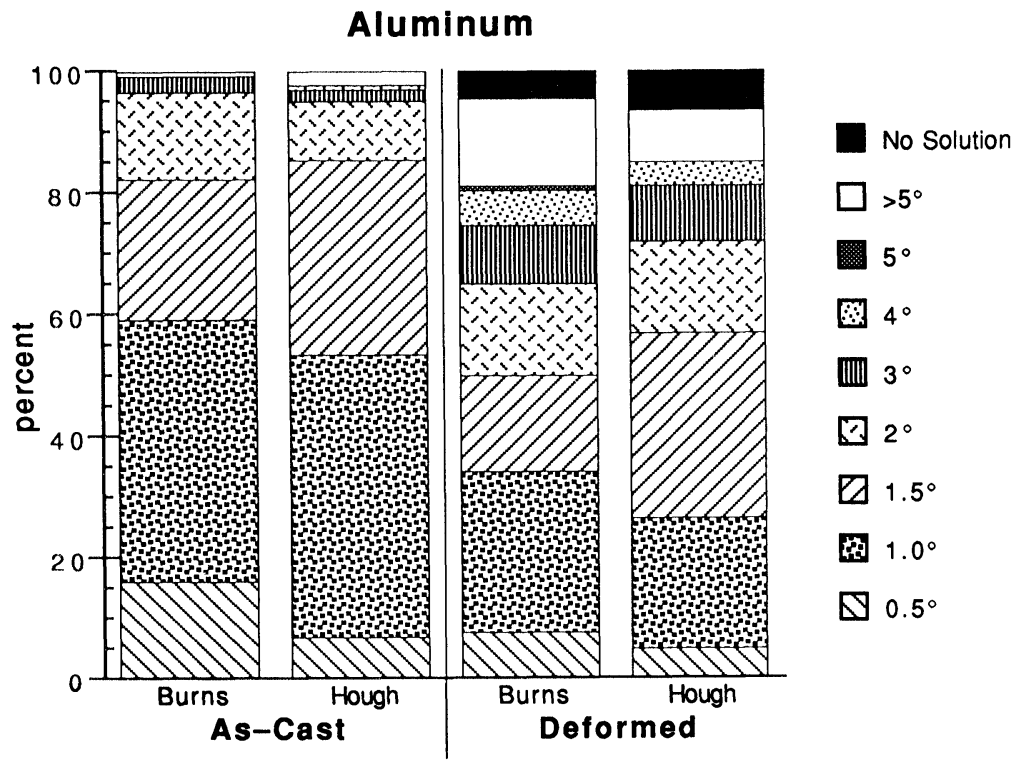

Figure 6 Orientation difference angle of different automatic indexing methods with reference to manual indexing of 120 patterns from as-cast aluminum and 106 patterns from deformed aluminum. 
Table 1 Summary of accuracy of automatic indexing methods.

\begin{tabular}{lllll}
\hline & $\begin{array}{l}\text { Burns } \\
\text { correct }\left(\omega<5^{\circ}\right)\end{array}$ & average $\omega$ & $\begin{array}{l}\text { Hough } \\
\text { correct }\left(\omega<5^{\circ}\right)\end{array}$ & average $\omega$ \\
\hline As-cast Al & $99.2 \%$ & $1.0^{\circ}$ & $97.5 \%$ & $1.0^{\circ}$ \\
Deformed Al & $81.1 \%$ & $1.6^{\circ}$ & $85.0 \%$ & $1.4^{\circ}$ \\
\hline
\end{tabular}

$\omega$ is the orientation difference angle between the automated and manual indexings.

\section{DISCUSSION}

\section{Hough vs. Burns}

As mentioned previously the Hough algorithm is more robust in the case of lower quality patterns, whereas the Burns algorithm is more accurate in the case of higher quality patterns. This is especially evident if one excludes the patterns for which no solutions were found. The problem with high quality patterns may be due, in part, to the detection of second order bands (such as the $\{133\}$ type band) which are not included in the look-up table at present. One way to avoid this problem is to tighten the tolerance of the interplanar angle in the look-up table searching procedure $\left(5^{\circ}\right.$ was used). This makes sense for the high quality patterns where the bands are more accurately detected.

In both methods an image quality parameter is recorded for each analyzed pattern, which is supposed to estimate contrast and sharpness of the bands in the EBSP. The Burns technique evaluates image quality by the total detected edge length $\left(\mathrm{IQ}_{\mathrm{B}}\right)$ as defined by Wright and Adams (1992). The Hough approach obtains a quality parameter $\left(\mathrm{IQ}_{\mathrm{H}}\right)$ from the sum of all selected peak magnitudes in Hough space as described previously. Because of these different definitions there is no exact relationship between both parameters. Nevertheless, comparison of the IQ's for the patterns from as-cast and deformed aluminum (Figure 7)

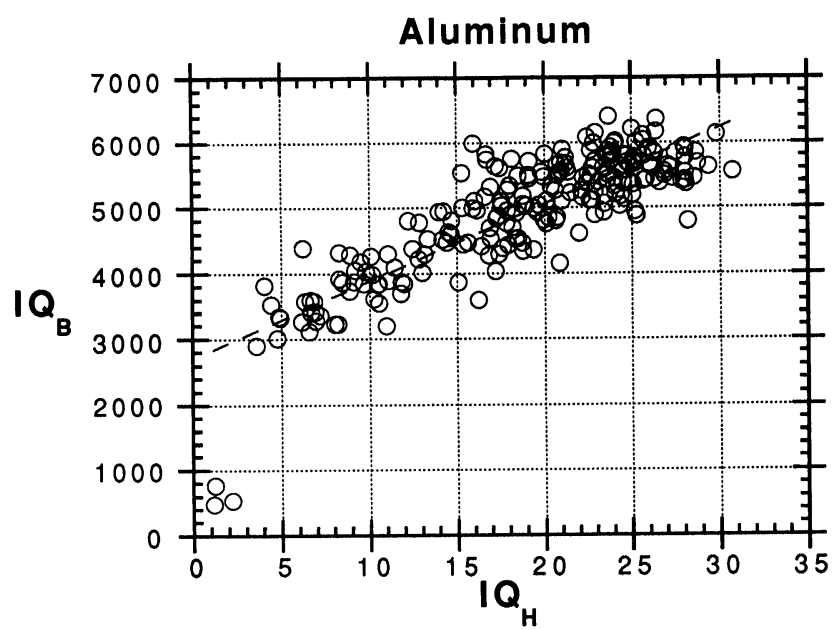

Figure 7 Correlation of image quality parameters of the Burns $\left(\mathrm{IQ}_{B}\right)$ and Hough $\left(\mathrm{IQ}_{H}\right)$ techniques (dashed: curve of linear best fit). Patterns are from as-cast and deformed aluminum. 

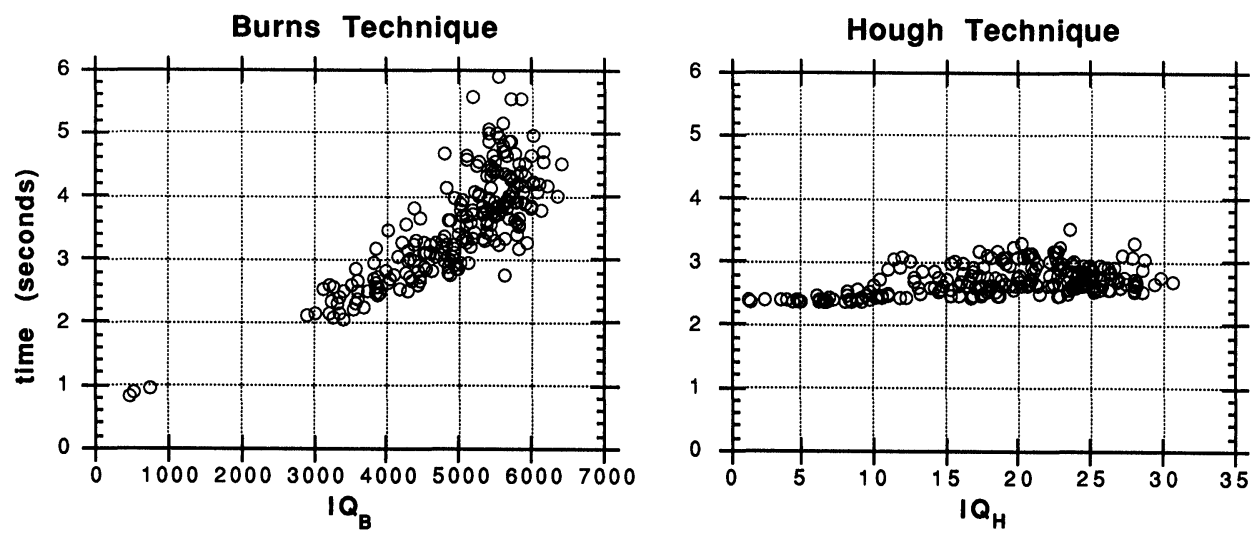

Figure 8 Pattern indexing time versus image quality parameter (IQ). Patterns are from as-cast and deformed aluminum.

shows some correlation, where the indicated linear Least Squares fit gives a regression parameter of 0.84 . It should be pointed out, that $\mathrm{IQ}_{\mathrm{H}}$ has a much broader interval of scattering and seems to be more selective than $\mathrm{IQ}_{\mathrm{B}}$.

Another issue of comparison is speed. As can be seen from Figure 8 , the Hough algorithm is faster than the Burns approach for higher quality patterns. The time spent in the Hough approach is also nearly constant for all patterns. This is because the majority of time (73\%) spent in the Hough approach is in calculating the Hough transform and its convolution which is the same procedure for all images. In the Burns approach, local edges are found which are correlated into bands. In higher quality patterns, more edges are found, resulting in more time being consumed in correlating the edges into bands. The Hough approach is also more suited to hard-coding since the transform is the same for every image and independent of crystallographic symmetry. At present the time spent in processing the image (averaging and background subtraction) and in determining the orientation from the pattern are nearly the same and are done in parallel.

\section{Precision}

In order to ascertain the effectiveness of the orientation averaging scheme (described earlier) in making the orientation measurements more precise, the copper patterns were automatically analyzed with and without orientation averaging included in the algorithms. The results as compared with the manual indexing are shown in Figure 5. The averaging slightly improves the precision.

The idea of precision has been discussed somewhat by Hjelen et al. (1991). We have found, however, that in practical use of EBSP the error of obtaining the absolute orientation is larger than $0.5^{\circ}$ claimed by Hjelen et al. The right bar in Figure 9 shows the comparison of orientations determined by two different and independent operators on the same set of patterns (the 120 as-cast aluminum patterns). The difference between measured orientations was as much as $4^{\circ}$ and the mean was $0.94^{\circ}$. Despite the close agreement, it cannot be inferred from Figure 9 that the automated indexing is more accurate than the manual indexing 


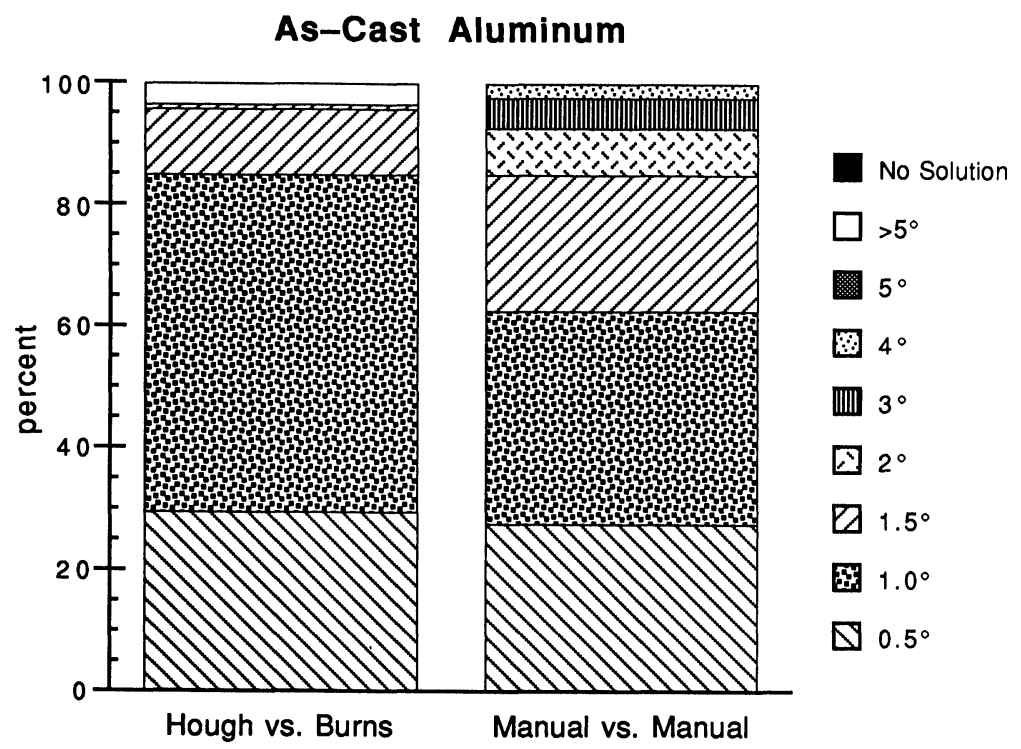

Figure 9 Orientation difference angle between two different automatic analyses and between two independent manual analyses for 120 patterns from as-cast aluminum.

since the true orientations are not known. However, a strong case can be made for the precision of automatic indexing as opposed to manual on high quality patterns. When a pattern is indexed manually, one user may select a zone axis pair from one side of the pattern and a second user may select axes from the opposite side. This magnifies any inaccuracies due to calibration to the fullest extent, especially when the digitized zone axes lie close together in the pattern. Bands detected automatically extend across the entire diffraction image. The orientation which best fits all of the detected bands is determined. This orientation minimizes the errors resulting from calibration.

\section{Reliability}

The primary needs for an automated indexing program are first to correctly identify as many patterns as possible and second to recognize patterns which have probably been incorrectly analyzed. The image quality parameter serves as an indicator of the likelihood that a pattern is correctly or incorrectly indexed. If a solution can not be found for the bands detected or if only two bands are found then the algorithm knows that the pattern can not be correctly indexed. However, spurious bands are sometimes found which lead to incorrect solutions. This is more likely to happen when the pattern is of poor quality as can be observed in Figure 10. A cut-off point in image quality can be used in both techiques to maximize the probability that a pattern has been correctly analyzed. For example, in Figure 10 nearly $100 \%$ reliability can be achieved by throwing out solutions obtained from patterns with $\mathrm{IQ}_{\mathrm{H}}$ less than 13 . Lower quality patterns can arise from a high dislocation density, lattice curvature, surface contamination, the probe being on a grain boundary or surface topology. However, there may be 

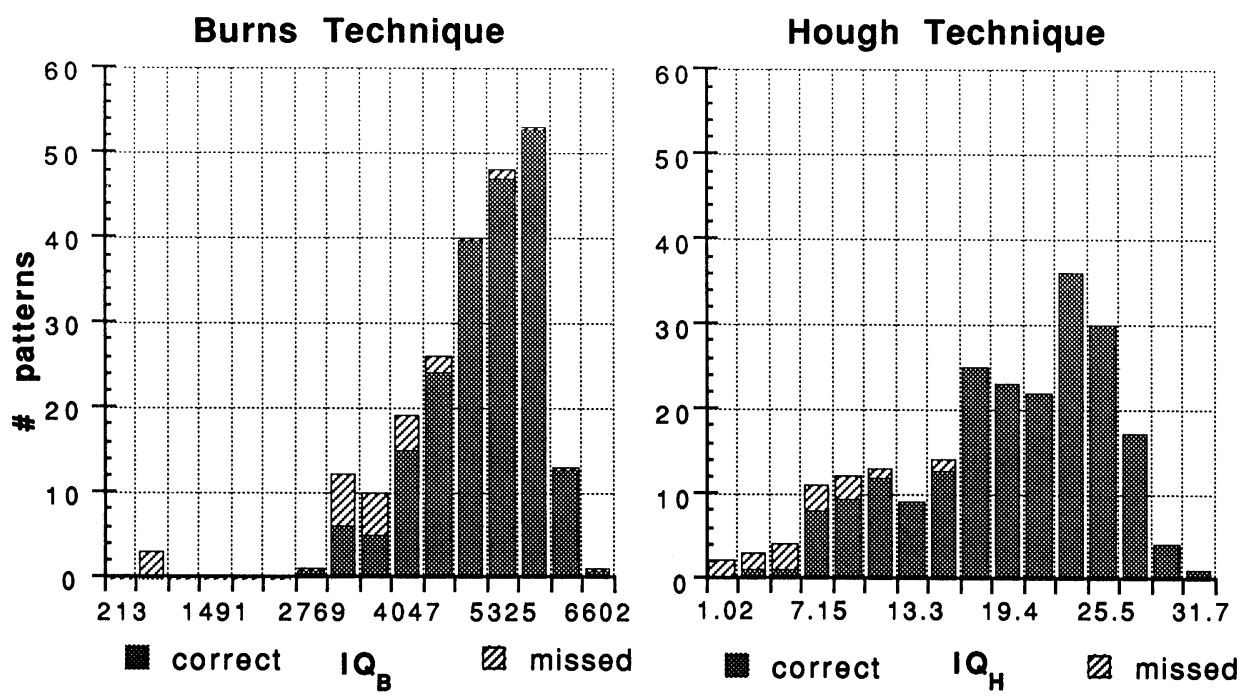

Figure 10 Number of patterns indexed correctly and incorrectly versus image quality parameter (IQ). Patterns are from as-cast and deformed aluminum.

cases when orientations from grains of high strain may be of primary interest. In this case it would not be prudent to simply throw out patterns of low image quality.

The more even distribution of $\mathrm{IQ}_{\mathrm{H}}$ over a wide interval may indicate another application of the Hough technique. Recent work by Wilkinson and Dingley (1991) has shown that it is possible to link the quality of images with the amount of plastic strain. Although Wilkinson and Dingley (1991) did not use the Hough transform in their work, a form of the $\mathrm{IQ}_{\mathrm{H}}$ presented here may be used to derive approximations of plastic strain levels in the material.

\section{CONCLUSIONS}

The addition of a computer controlled $x-y$ stage into the SEM enables a system for collecting single orientation measurements to be designed which can be operated without any direct operator intervention during the measurement.

The Hough transform and the Burns algorithm both provide a reliable means for rapidly obtaining single orientation measurements through band detection in EBSPs. There appears to be only a slight difference in the ability of the two approaches to correctly identify the bands. The Hough method has the advantage of being somewhat material independent (the optimum butterfly mask is material dependent). The Burns algorithm is faster for lower quality patterns, whereas the Hough is faster for higher quality patterns. However, the Hough algorithm is slightly more reliable for lower quality patterns and the Burns is slightly more reliable for the higher quality patterns. Thus, the choice of which algorithm to use should be based on the average quality of patterns to be analyzed while recognizing the tradeoffs of speed and reliability. Both techniques have para- 
meters which are material and/or camera geometry dependent. In order to optimize these parameters for a given material or camera geometry, it is necessary to do some of the analysis that we have shown here.

\section{Acknowledgements}

This work was supported by a Materials Research Group Award from the National Science Foundation (DMR-9001378).

\section{References}

Burns, J. B., Hanson, A. R. and Riseman, E. M. (1986). Extracting Straight Lines. IEEE Transactions on Pattern Analysis and Machine Intelligence, 8, 425-455.

Dingley, D. J. and Baba-Kishi, K. (1986). Use of Electron Back Scatter Diffraction Patterns for Determination of Crystal Symmetry Elements. Scanning Electron Microscopy, II, 383-391.

Hjelen, J., Ørsund, R., Hoel, E., Runde, P., Furu, T. and Nes, E. (1991). EBSP, Progress in Technique and Applications, presented at the joint TMS/ASM October meeting.

Illingworth, J. and Kittler, J. (1988). A Survey of the Hough Transform. Computer Vision, Graphics and Image Processing, 44, 87-116.

Kunze, K. (1991). Zur quantitativen Texturanalyse von Gesteinen: Bestimmung, Interpretation und Simulation von Quarzteilgefügen. Dissertation RWTH Aachen, ISSN 0514-8790, 12-26.

Lassen, N. C. K., Conradsen, K. and Juul-Jensen, D. (1992). Image Processing Procedures for Analysis of EBSPs. submitted to Scanning Microscopy International.

Leavers, V. F. and Boyce, J. F. (1987). The Radon Transform and its Application to Shape Parameterization in Machine Vision. Image and Vision Computing, 5, 161-166.

Morawiec, A. and Pospiech, J. (1989). Some Information of Quaternions Useful in Texture Calculations. Texture and Microstructures, 10, 211-216.

Venables, J. A. and Harland, C. J. (1973). Electron Backscatter Patterns-A New Technique for obtaining Crystallographic Information in the SEM. Philosophical Magazine, 27, 1193-1200.

Wilkinson, A. J. and Dingley, D. J. (1991). Quantitative Deformation Studies Using Electron Back Scatter Patterns. Acta Metallurgica et Materialia, 39, 3047-3055.

Wright, S. I. and Adams, B. L. (1992). Automatic Analysis of Electron Backscatter Diffraction Patterns. Metallurgical Transactions, 23A, 759-767. 